\title{
Measurements of the local convective heat flux in turbulent Rayleigh-Bénard convection
}

\author{
X.-D. Shang, ${ }^{1,2}$ X.-L. Qiu, ${ }^{1}$ P. Tong, ${ }^{1,3}$ and K.-Q. Xia ${ }^{2}$ \\ ${ }^{1}$ Department of Physics, Oklahoma State University, Stillwater, Oklahoma 74078, USA \\ ${ }^{2}$ Department of Physics, the Chinese University of Hong Kong, Shatin, Hong Kong \\ ${ }^{3}$ Department of Physics, Hong Kong University of Science and Technology, Clear Water Bay, Kowloon, Hong Kong
}

(Received 26 January 2004; published 30 August 2004)

\begin{abstract}
A systematic study of the local convective heat transport in turbulent thermal convection is carried out in small-aspect-ratio cells filled with water. The local convective heat flux is obtained from the simultaneous velocity and temperature measurements over varying Rayleigh numbers and spatial positions across the entire convection cell. Large fluctuations of the local convective heat flux are found mainly in the vertical direction and they are determined primarily by the thermal plumes in the system. The experiment reveals the spatial distribution of the local convective heat flux in a closed cell and thus settles a long-debated issue on how heat is transported in small-aspect-ratio cells.
\end{abstract}

DOI: 10.1103/PhysRevE.70.026308

PACS number(s): 47.27.Te, 44.25.+f, 47.27.-i

\section{INTRODUCTION}

An intriguing feature of turbulent Rayleigh-Bénard convection is the emergence of a coherent large-scale circulation (LSC), which spans the height of the convection cell [1-4]. This coherent flow structure arises in a turbulent environment with irregular bursts of thermal plumes from the upper and lower thermal boundary layers. In an attempt to further understand the structure and dynamics of the thermal plumes, we have recently carried out a series of independent temperature and velocity measurements in turbulent thermal convection [5-9]. Using the techniques of laser Doppler velocimetry (LDV), thermometry, and flow visualization, we mapped out the temperature and velocity fields in the plane of LSC. The temperature and velocity measurements were conducted in convection cells with different aspect ratios and over varying Rayleigh numbers and spatial positions across the entire cell. Water was used as the convecting fluid, in which both the temperature and velocity measurements can be made with high accuracy. These local measurements provided a body of reliable velocity and temperature data and complemented the global measurements of heat transport in turbulent convection [1,10-15].

An important finding of these experiments is that the spatial distribution of thermal plumes in a closed convection cell is neither homogeneous nor isotropic. The thermal plumes organize themselves in such a way that warm plumes accumulate on one side of the cell and cold plumes concentrate on the opposite side of the cell. The warm and cold plumes, which are separated laterally in the two opposing sidewall regions, exert buoyancy forces on the fluid and drive the vertical flow near the sidewall. The central core region is "sheared" by the rising and falling plumes, resulting in a large-scale circulation across the cell height. This coherent quasi-two-dimensional flow structure is superimposed on a turbulent background with large velocity fluctuations both in the central region and near the cell boundary.

An important question one might ask is how the spatial organization of the thermal plumes and the large-scale flow structure affect the heat transport in turbulent thermal convection. A large number of global heat transport measure- ments have been carried out in various convecting fluids and under different experimental conditions $[1-4,16]$. Some of the measurements were conducted with a wide parameter range and great precession [1,10-15]. These measurements have stimulated considerable theoretical efforts aimed at explaining the functional form of the measured Nusselt number (normalized total heat flux) $\mathrm{Nu}(\mathrm{Ra}, \mathrm{Pr}$ ) as a function of two experimental control parameters: the Rayleigh number Ra and the Prandtl number Pr. Like many transport phenomena in condensed matter physics, the measured macroscopic transport properties can often be explained by theories with different microscopic mechanisms [1-4]. A main issue of an unresolved theoretical debate is whether the heat transport in turbulent convection is determined primarily by thermal plumes or by the large-scale circulation. Direct measurements of the local heat flux, therefore, become essential to the understanding of the heat transport mechanism in turbulent convection.

In this paper, we report simultaneous measurements of the local velocity $\mathbf{v}(\mathbf{r}, t)$ and the local temperature $T(\mathbf{r}, t)$, from which we obtain the local convective heat flux $[17,18]$

$$
\mathbf{J}(\mathbf{r})=\frac{\left\langle\mathbf{v}(\mathbf{r}, t) \delta T_{0}(\mathbf{r}, t)\right\rangle_{t} H}{\kappa \Delta T},
$$

where $\kappa$ is the thermal diffusivity of the convecting fluid, $\Delta T$ is the temperature difference across the convection cell of height $H$, and $\langle\cdots\rangle_{t}$ represents a time average over $t$. The local temperature fluctuation is defined as $\delta T_{0}(\mathbf{r}, t)=T(\mathbf{r}, t)$ $-T_{0}$ with $T_{0}$ being the mean temperature of the bulk fluid. As indicated by Eq. (1), the local convective flux $\mathbf{J}(\mathbf{r})$ is normalized by the conductive heat flux $\kappa \Delta T / H$. In the experiment, we measure $\mathbf{J}(\mathbf{r})$ over varying Rayleigh numbers and spatial position $\mathbf{r}$ across the entire cell. These measurements reveal how heat is transported vertically out of the thin thermal boundary layers near the upper and lower conducting surfaces. It is found that the heat transport in turbulent convection over the parameter range studied is carried out mainly by the thermal plumes. The experiment shows that the heat transport in the aspect-ratio-1 cell takes place primarily 
along the cell periphery in the direction of the large-scale circulation.

The remainder of the paper is organized as follows. We first describe the apparatus and the experimental method in Sec. II. Experimental results are discussed in Sec. III. Finally, the work is summarized in Sec. IV.

\section{EXPERIMENT}

The experiment is conducted in two upright cylindrical cells filled with water. The two convection cells have the same inner diameter of $D=19.0 \mathrm{~cm}$ but their height is different; one is $20.5 \mathrm{~cm}$ and the other is $40.9 \mathrm{~cm}$. The corresponding aspect ratio $(A=$ diameter $D /$ height $H)$ of the two cells is, respectively, $A \simeq 1$ and $A \simeq 0.5$. Details about the apparatus have been described elsewhere [16,19], and here we mention only some key points. The sidewall of the cells is made of a transparent Plexiglas ring with wall thickness $0.6 \mathrm{~cm}$. The top and bottom plates are made of brass and their surfaces are electroplated with a thin layer of gold. The thickness of the top plate is $1.0 \mathrm{~cm}$ and that of the bottom plate is $0.85 \mathrm{~cm}$. The Plexiglas ring is sandwiched between the two plates and is sealed to the top and bottom plates via two rubber $\mathrm{O}$ rings. Two silicon rubber film heaters connected in parallel are sandwiched on the backside of the bottom plate to provide constant and uniform heating. A dc power supply with $99.99 \%$ long-term stability is used to provide the heating power. The voltage applied to the heaters varies from 20 to $80 \mathrm{~V}$, and the corresponding heating power is in the range between $23 \mathrm{~W}$ and $371 \mathrm{~W}$. The upper side of the top plate together with a circular aluminum cover form a closed cooling chamber, whose temperature is maintained constant by circulating cold water from a temperaturecontrolled bath and circulator. The temperature stability of the circulator is $0.01^{\circ} \mathrm{C}$. The temperature difference $\Delta T$ between the top and bottom plates is measured by two thermistors embedded in each plate.

The two cells are used to change the aspect ratio and to extend the accessible range of the Rayleigh number Ra $=\alpha g \Delta T H^{3} /(\nu \kappa)$, where $g$ is the gravitational acceleration, $H$ is the cell height, and $\alpha, \nu$, and $\kappa$ are, respectively, the thermal expansion coefficient, the kinematic viscosity, and the thermal diffusivity of the convecting fluid (water). The numerical values of the fluid parameters are taken from the CRC Handbook of Chemistry and Physics [20]. The A=1 cell covers the Ra range between $9.8 \times 10^{8}$ and $7.6 \times 10^{9}$. In the $A=0.5$ cell the maximum value of $\mathrm{Ra}$ can reach up to $5.9 \times 10^{10}$. In the experiment, the temperature difference $\Delta T$ between the top and bottom plates varies from $4.8^{\circ} \mathrm{C}$ to $35^{\circ} \mathrm{C}$ depending on the heating power. By adjusting the temperature of the cooling water, we maintain the temperature of the bulk fluid at $\sim 29^{\circ} \mathrm{C}$ for all the measurements. At this temperature, one has $\nu \simeq 8.2 \times 10^{-3} \mathrm{~cm}^{2} / \mathrm{s}, \quad \kappa \simeq 1.5$ $\times 10^{-3} \mathrm{~cm}^{2} / \mathrm{s}$, and the corresponding Prandtl number $\operatorname{Pr}$ $=\nu / \kappa \simeq 5.5$. The temperature stability of the top and bottom plates is found to be within $0.1{ }^{\circ} \mathrm{C}$ in standard deviation, which is less than $2.1 \%$ of the minimum $\Delta T$ used in the experiment.

Figure 1(a) shows the experimental arrangement for the simultaneous velocity and temperature measurements. Local
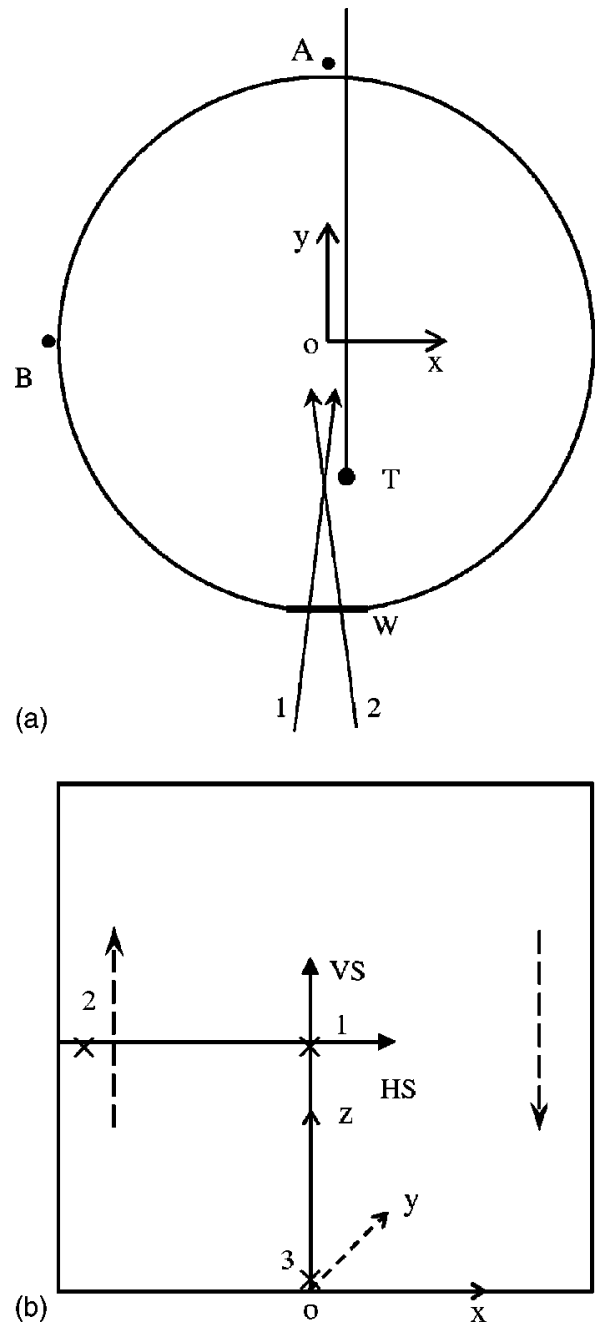

FIG. 1. (a) Schematic diagram of the experimental setup (top view): T, temperature probe; 1,2 , two laser beams for LDV; W, optical window. (b) Space coordinates used for the presentation of the local convective heat flux measurements. The long horizontal (HS) and vertical (VS) arrows indicate, respectively, the tracks of the horizontal and vertical scans of the simultaneous velocity and temperature measurements. The three crosses marked with a number indicate the positions used for the statistical study of local flux fluctuations. The dashed arrows near the sidewall indicate the direction of the large-scale circulation.

velocity measurements are conducted using a twocomponent LDV system (TSI Inc.) together with an argonion laser (Coherent Innova 90). A long rectangular flat window (W) made of transparent Plexiglas with wall thickness $0.6 \mathrm{~cm}$ is inserted onto the sidewall to admit the incident laser beams and observe the scattered light by seed particles. The width of the optical window is $3 \mathrm{~cm}$ and the length remains the same as the height of the Plexiglas ring. The use of the flat window eliminates the optical distortions generated by the curvature of the sidewall and thus increases the LDV data rate, especially in the central region of the cell. Two pairs of laser beams of different colors (blue and green) coming from a LDV fiber-optic transceiver are directed through the optical window and focused onto a single point inside the convection cell. The fiber-optic transceiver has a 
receiving fiber, which collects the scattered light in the backward direction and feeds it to two photomultiplier tubes. One of the photomultiplier tubes detects the blue light and the other detects the green light. The laser focusing spot has a cylindrical probe volume of $1.31 \mathrm{~mm}$ in length and $0.09 \mathrm{~mm}$ in diameter. The two laser beams shown in Fig. 1(a) are in the horizontal plane and are used to measure the horizontal velocity $v_{x}$ in the $x$ direction. The other two laser beams (not shown) are in the vertical plane parallel to the propagation direction of the laser beams. These two beams are used to measure the vertical velocity $v_{z}$ along the $z$ axis.

Monodisperse polymer latex spheres of $4.75 \mu \mathrm{m}$ in diameter are used as seed particles. Because their density $\left(1.05 \mathrm{~g} / \mathrm{cm}^{3}\right)$ closely matches that of water, the seed particles follow the local flow well. With the LDV arrangement shown in Fig. 1(a), we measure the local velocity as a function of time, and the measuring position (laser focusing spot) can be varied continuously along the $y$ axis (horizontal scan) and the $z$ axis (vertical scan). This is accomplished by moving the fiber-optic transceiver, which is mounted on a traversable table. It has been shown $[5,6]$ that LDV is capable of measuring the flow velocity with high accuracy (better than $1 \%$ ) over the entire convection cell, except near the upper and lower thermal boundary layers, whose thickness is $\sim 1 \mathrm{~mm}$ at $\mathrm{Ra} \simeq 10^{9}$.

A typical sampling rate of the velocity measurements is $10-15 \mathrm{~Hz}$, which is approximately $3-10$ times (depending on the value of $\mathrm{Ra}$ ) larger than the cutoff frequency of the velocity fluctuations in the system. Typically, we take 2-h-long time series data $\left(\sim 10^{5}\right.$ data points) at each spatial position for the profile measurements and 7-h-long time series data $\left(\sim 4 \times 10^{5}\right.$ data points) for further statistical analysis. This data accumulation time is much longer than the time scale associated with the large-scale circulation, which is of the order of $1 \mathrm{~min}$ at $\mathrm{Ra} \simeq 3 \times 10^{9}$, ensuring that the statistical average of flow properties is adequate. In this way, we obtain a complete series of velocity data at various Rayleigh numbers and spatial positions with the highest statistical accuracy possible. In the calculation of the velocity statistics, we use the transit time weighting to correct for the velocity sampling bias [21].

Temperature measurements are simultaneously taken using a multichannel LDV interface module (TSI Datalink) for synchronization of the data acquisition. A triggering pulse from the LDV signal processor initiates the acquisition of an analog temperature signal. A small movable thermistor $(\mathrm{T})$ of $0.2 \mathrm{~mm}$ in diameter, $15 \mathrm{~ms}$ in response time, and $20 \mathrm{mK} / \Omega$ in temperature sensitivity (Thermometrics, AB6E3B05KA202R) is used to measure the local fluid temperature $T(\mathbf{r}, t)$. All the thermistors used in the experiment are calibrated individually with an accuracy of $0.01^{\circ} \mathrm{C}$. To guide the thermistor into the cell, we install a horizontal stainless steel tube on the sidewall opposite the optical window. The stainless steel tube (Type 304 SS hypodermic tubing) has an outer diameter $1.07 \mathrm{~mm}$ and wall thickness $0.19 \mathrm{~mm}$ and is mounted at the midheight of the cell. Thin thermistor wires thread through the stainless steel tube from the outside and a small head piece of the sensor tip sticks out of the tube end inside the convection cell. The tube end together with the sensor tip is sealed with glue so that the convecting fluid cannot leak out through the stainless steel tube. The tube can slide in and out so that $T(\mathbf{r}, t)$ can be measured at various horizontal positions along the cell diameter (horizontal scan).

Similarly, we also install a vertical stainless steel tube through the center of the top plate and measure $T(\mathbf{r}, t)$ along the central vertical axis of the cell (vertical scan). In the experiment, we place the thermistor at a position very close to the laser focusing spot. The spatial separation between the laser focusing spot and the thermistor tip is fixed and we will discuss how the separation is chosen in the next section. The thermistor is connected to an ac bridge as a resistance arm. The voltage signals from the ac bridge are fed to the interface module, whose output is connected to the LDV signal processor. A host computer is used to store each pair of velocity and temperature measurements with the same time stamp. Because the measurements of the two velocity channels are conducted independently, which allows a higher sampling rate compared with coincident measurements between the two velocity channels, the temperature time series has approximately twice as many data points as that of each velocity component. To facilitate the calculation of the power spectrum and the correlation function, which requires evenly spaced time series data, both the temperature and velocity time series are extrapolated linearly according to the average sampling rate of the temperature measurements. Calculations of the local convective heat flux are also made using the extrapolated time series data. The use of the evenly spaced time series data helps to correct for the velocity sampling bias of unevenly sampled LDV data [21].

Figure 1(b) shows the space coordinates to be used below in the presentation of the local convective heat flux measurements. The origin of the coordinate system is chosen to coincide with the center of the lower conducting surface. The $x$ and $z$ axes are in the rotation plane of the LSC and the $y$ axis is perpendicular to the rotation plane. The long horizontal (HS) and vertical (VS) arrows indicate, respectively, the tracks of the horizontal and vertical scans of the simultaneous velocity and temperature measurements. The three crosses shown in Fig. 1(b) indicate the positions used for the study of the statistical properties of local convective heat flux fluctuations. Early temperature measurements[22] showed that the azimuth of LSC rotates slowly in time when the cylindrical cell is leveled perfectly. It was found recently that the LSC in the leveled cylindrical cell also reverses its rotational direction randomly $[23,24]$.

To pin down the azimuthal rotation and the random reversal of the LSC, we tip the cell with a small angle $\left(<1^{\circ}\right)$ by adding a few sheets of paper on one side of the cell bottom. Ciliberto et al. [25] have shown that such a small tilt does not affect turbulent convection very much. When we tip the cell at position B [see Fig. 1(a)], the LSC is set up in the $x$ $-z$ plane and the direction of rotation is shown by the two long-dashed arrows in Fig. 1(b). In this case, the LDV setup shown in Fig. 1(a) is capable of measuring the two in-plane velocity components $v_{x}$ and $v_{z}$. One can also tip the cell at position A and set up LSC in the $y-z$ plane. In this case, we can measure the in-plane velocity component along the mean flow direction and the out-of-plane velocity component perpendicular to the rotation plane of the LSC. To avoid confusion, hereinafter we choose the $x-z$ plane to coincide with the 

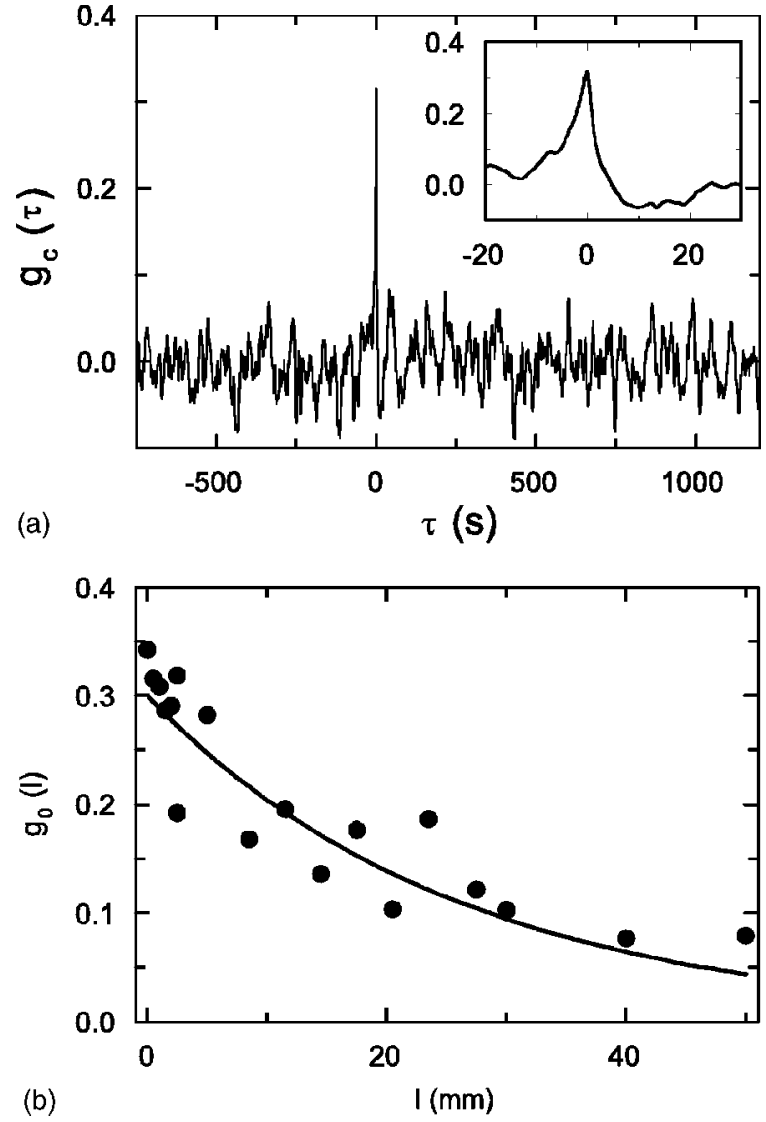

FIG. 2. (a) Measured velocity-temperature cross-correlation function $g_{c}(\tau)$ as a function of delay time $\tau$ at $\mathrm{Ra}=3.6 \times 10^{9}$. In the measurement, both the velocity and temperature probes are placed at the cell center and their separation is $\ell=0.5 \mathrm{~mm}$. The inset shows the same $g_{c}(\tau)$ with smaller range of $\tau$ around $\tau \simeq 0$. (b) Measured correlation amplitude $g_{0}(\ell)$ as a function of $\ell$. The solid curve shows the fitted function $g_{0}(\ell)=0.3 \exp (-\ell / \xi)$, with $\xi=26 \mathrm{~mm}$.

rotation plane of the LSC regardless of the actual arrangement of the tilt.

\section{RESULTS AND DISCUSSION}

\section{A. Measurement of the local convective heat flux}

According to Eq. (1), the local convective heat flux $\mathbf{J}(\mathbf{r})$ is obtained from simultaneous measurements of the local velocity $\mathbf{v}(\mathbf{r}, t)$ and the local temperature $T(\mathbf{r}, t)$ at the same position $\mathbf{r}$. In the experiment, however, we have to measure $\mathbf{v}(\mathbf{r}, t)$ and $T(\mathbf{r}, t)$ at two different positions with a small separation $\ell$, so that the thermistor tip does not block the laser beams. This separation should be small compared with the correlation length $\xi$ between the temperature and velocity fluctuations. On the other hand, it should be large enough to minimize the disturbances produced by the thermistor tip to the velocity measurement. Figure 2(a) shows the velocitytemperature cross-correlation function $g_{c}(\tau)=\left\langle\delta v_{z}(t) \delta T(t\right.$ $+\tau)\rangle /\left(v_{r m s} T_{r m s}\right)$, as a function of delay time $\tau$ measured at the cell center. Here the temperature fluctuation is defined as $\delta T(t)=T(t)-\bar{T}$, with $\bar{T}$ being the local mean value of $T(t)$.
Similarly, the vertical velocity fluctuation is defined as $\delta v_{z}(t)=v_{z}(t)-\bar{v}_{z}$, where $\bar{v}_{z}(\simeq 0$ at the cell center $)$ is the local mean value of $v_{z}(t)$. The cross-correlation function is normalized by the product of the velocity and temperature standard deviations, $v_{r m s}$ and $T_{r m s}$. It is found that the measured $g_{c}(\tau)$ has a single peak at $\tau \simeq 0$. The amplitude of the peak is $g_{0} \equiv g_{c}(0) \simeq 0.3$, which is much larger than the background noise at the level of 0.05 .

From the inset of Fig. 2(a), we find that the full width at half maximum (FWHM) of the peak is $\Gamma_{V T} \simeq 5.6 \mathrm{~s}$. The corresponding autocorrelation functions of the temperature and velocity fluctuations themselves (not shown) have a similar shape to the cross-correlation function and their FWHM values are, respectively, $\Gamma_{T} \simeq 2.4 \mathrm{~s}$ and $\Gamma_{V} \simeq 5.5 \mathrm{~s}$ at $\mathrm{Ra}=3.6$ $\times 10^{9}$. Clearly, the FWHM value of the measured $g_{c}(\tau)$ is determined by the larger peak width $\Gamma_{V}$. The half peak width $\Gamma_{T} / 2$ of the temperature autocorrelation function provides information about the transient time, $\Gamma_{t} / 2 \simeq \delta_{p} / U$, for the temperature fluctuations of characteristic size $\delta_{p}$ ("vertical plume size") passing through the temperature probe with a speed $U$ [8]. At $\mathrm{Ra}=3.6 \times 10^{9}$, we have $U \simeq U_{r m s}$ $\simeq 3.3 \mathrm{~mm} / \mathrm{s}$ and thus $\delta_{p} \simeq 3.9 \mathrm{~mm}$. This value of $\delta_{p}$ is slightly larger than the average thermal boundary layer thickness $\bar{\delta}(\simeq 1 \mathrm{~mm})$ at the same Ra[26]. Figure 2(a) thus suggests that the correlated fluctuations are localized events and occur mostly in small time intervals (i.e., in the form of "plumes") even at the cell center. Similar cross-correlation functions are also observed near the sidewall.

Figure 2(b) shows how the measured $g_{0}$ varies with the separation $\ell$ between the LDV focusing spot and the thermistor tip. Because the convective flow is slow and the time average runs for less than an hour for these measurements, the $g_{0}(\ell)$ obtained has somewhat larger scatter. Nevertheless, it is clearly shown that $g_{0}(\ell)$ decreases with increasing $\ell$, and the decay may be described by a simple exponential function $g_{0}(\ell)=0.3 \exp (-\ell / \xi)$, with $\xi=26 \mathrm{~mm}$ (solid curve). A similar correlation length is also found in the sidewall region. From these measurements we decided to keep the separation at a minimal value $\ell_{0}=0.7 \pm 0.2 \mathrm{~mm}$. This distance is three times larger than the tip diameter of the thermistor but 37 times smaller than the correlation length $\xi$. It is comparable to the thermal boundary layer thickness $\delta(\simeq 0.8 \mathrm{~mm}$ at Ra $\left.=3.6 \times 10^{9}\right)$, which is the smallest length scale in turbulent convection. For the measurements in the sidewall region, the thermistor is always placed at the downstream position to further minimize the disturbances produced by the temperature probe.

Another issue in the calculation of $\mathbf{J}(\mathbf{r})$ is the choice of the reference temperature $T_{0}$ used in the definition of the temperature fluctuation $\delta T_{0}(\mathbf{r}, t)=T(\mathbf{r}, t)-T_{0}$. The value of $T_{0}$ should remain the same across the entire body of the convecting fluid. Figure 3(a) compares the temperature histograms $H\left(\delta T_{0}\right)$ measured at the cell center (circles) and near the sidewall (triangles). Here we took the most probable temperature as $T_{0}$, which is determined from the peak position of the measured $H\left(\delta T_{0}\right)$. Because temperature fluctuations at the cell center are symmetric, the value of $T_{0}$ is the same as the local average temperature $\bar{T}$. In the plume-dominated sidewall region, however, temperature fluctuations become 


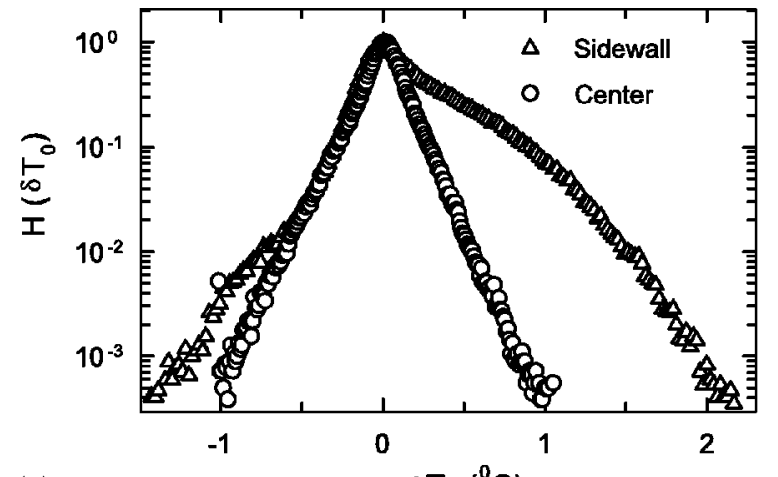

(a)

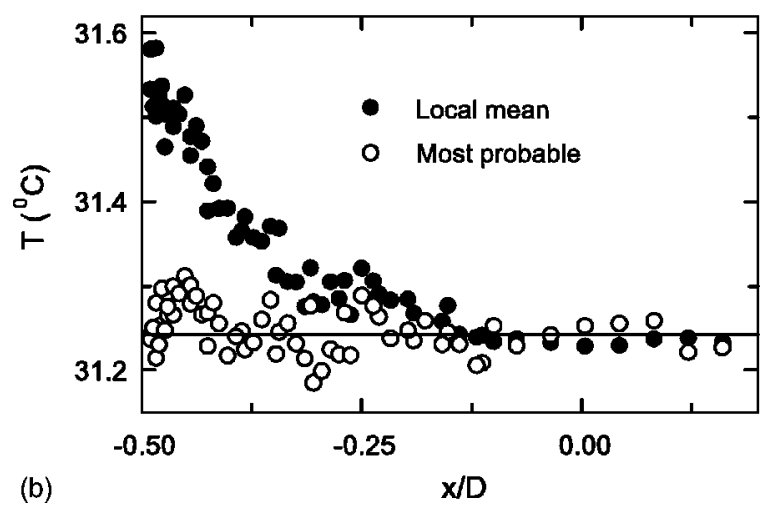

FIG. 3. (a) Measured histograms of the temperature fluctuations at the cell center (circles) and near the sidewall (triangles). (b) Profiles of the local average temperature $\bar{T}$ (solid circles) and the most probable temperature $T_{0}$ (open circles) as a function of $x / D$. The solid line is an average baseline for $T_{0}$. All the measurements are made in the $A=1$ cell at $\mathrm{Ra}=6.0 \times 10^{9}$.

highly skewed toward one direction and the measured $H\left(\delta T_{0}\right)$ shows a long decay tail. In this case, $T_{0}$ is no longer equal to $\bar{T}$. In addition to the numerical difference, the measured $\bar{T}$ also shows considerable spatial variations.

Figure 3(b) shows the horizontal profiles of the measured $\bar{T}$ (solid circles) and $T_{0}$ (open circles) in the midplane of the cell as a function of the normalized horizontal position $x / D$. It is seen that the local average temperature $\bar{T}$ remains constant only in the central region and gradually increases by more than $0.3^{\circ} \mathrm{C}$ when moved to the sidewall region. The value of $\bar{T}$ reaches maximum near the sidewall, where warm plumes concentrate. It has been shown [6] that on the opposite side of the cell cold plumes accumulate and $\bar{T}$ reaches a minimum value instead. The most probable temperature $T_{0}$, on the other hand, is found to remain on a constant baseline (solid line) across the entire body of the convecting fluid. Therefore, we choose the most probable temperature as the reference temperature in the calculation of $\mathbf{J}(\mathbf{r})$. It is seen from Fig. 3(b) that the experimental uncertainties for $T_{0}$ are within $\pm 0.05^{\circ} \mathrm{C}$, which are caused mainly by ambient temperature drifts during the measurements over a time period of several weeks. It should be pointed out that, while the dynamic equations for turbulent convection are invariant against a constant temperature shift, the actual measurement of $T(\mathbf{r}, t)$ is conducted at a given $T_{0}$. For a different value of
$T_{0}$, the corresponding $T(\mathbf{r}, t)$ will be different so that the resulting temperature fluctuations $\delta T_{0}(\mathbf{r}, t)=T(\mathbf{r}, t)-T_{0}$ remain unchanged (for fixed values of $\mathrm{Ra}$ and $\mathrm{Pr}$ ). Therefore, in the actual experiment one has to determine the reference temperature $T_{0}$ accurately in order to obtain the correct absolute value of $\mathbf{J}(\mathbf{r})$.

In addition to the convective contribution, the local heat flux also contains a diffusive contribution [18]

$$
\mathbf{J}_{d}(\mathbf{r})=-\frac{\boldsymbol{\nabla}\langle T(\mathbf{r}, t)\rangle_{t} H}{\Delta T},
$$

where $\nabla\langle T(\mathbf{r}, t)\rangle_{t}$ is the time-averaged temperature gradient at position $\mathbf{r}$, and the local diffusive flux $\mathbf{J}_{d}(\mathbf{r})$ defined in Eq. (2) is also normalized by the conductive heat flux $\kappa \Delta T / H$. The maximum temperature gradient occurs near the upper and lower thermal boundary layers, in which one finds $\nabla\langle T(z=0)\rangle_{t} \simeq \partial\langle T\rangle /\left.\partial z\right|_{\text {wall }} \simeq-\Delta T /(2 \delta)$ and thus $\left.\left(J_{d}\right)_{z}\right|_{\text {wall }}$ $\simeq H /(2 \delta)$ [18]. It was shown [26] that at $\mathrm{Ra}=3.6 \times 10^{9}, \delta$ $\simeq 0.8 \mathrm{~mm}$, and thus the corresponding $\left.\left(J_{d}\right)_{z}\right|_{\text {wall }} \simeq 128$ [27]. As mentioned above, the thermal boundary layer occupies only a thin region near the conducting surfaces and the turbulent bulk region is essentially isothermal [16,18]. Because of the lateral separation of warm and cold plumes, a small temperature gradient was indeed found in the $x$ direction [6]. At $\mathrm{Ra}=3.3 \times 10^{9}$, we have $\partial\langle T\rangle /\left.\partial x\right|_{\text {bulk }} \simeq 1.3 \mathrm{mK} / \mathrm{mm}$ and thus $\left.\left(J_{d}\right)_{x}\right|_{\text {bulk }} \simeq 1.6 \times 10^{-2}$ (for $\Delta T=17^{\circ} \mathrm{C}$ ). This is a negligibly small number compared with the local convective heat flux in the bulk region. In the following, we focus exclusively on the local convective heat flux (local heat flux or local flux for short) unless otherwise specified.

\section{B. Spatial distribution of the local convective heat flux}

$$
\text { 1. } A=1 \text { cell }
$$

We now discuss the measurements of $\mathbf{J}(\mathbf{r})$ in the rotation plane of the LSC. Some of the results have been reported briefly in a Letter [28]. For the horizontal scan (HS) shown in Fig. 1(b), we measure the vertical flux $J_{z}(x)$ and the horizontal flux $J_{y}(x)$ out of the rotation plane. For the vertical scan (VS), we can measure all three flux components $J_{x}(z)$, $J_{y}(z)$, and $J_{z}(z)$. Figure $4\left(\right.$ a) shows the measured $J_{z}(x)$ (solid circles) and $J_{y}(x)$ (open circles) as a function of the normalized horizontal position $x / D$ at $\mathrm{Ra}=3.6 \times 10^{9}$. It is seen that the heat flux across the midplane of the cell is predominantly in the vertical direction and the horizontal heat flux is negligible. The vertical heat flux is concentrated in the sidewall region. It is found that the amplitude of the vertical flux profile $J_{z}(x)$ increases with $\mathrm{Ra}$ but its shape remains unchanged in the Ra range studied. Figure 4(b) displays the normalized $J_{z}(x)$ by its peak value $J_{0}$ at three different values of Ra. It is seen that all the measured $J_{z}(x) / J_{0}$ can be superposed on a single master curve. The vertical flux at the cell center is small but is definitely nonzero. It accounts for approximately $5 \%$ of the peak value $J_{0}$ near the sidewall. Using the estimated uncertainty of $0.05^{\circ} \mathrm{C}$ for $\delta T_{0}$, we calculate the error bar for $J_{z}(x)$. It is seen from Fig. 4(a) that the measured $J_{z}(x)$ near the sidewall has a relatively larger error bar com- 

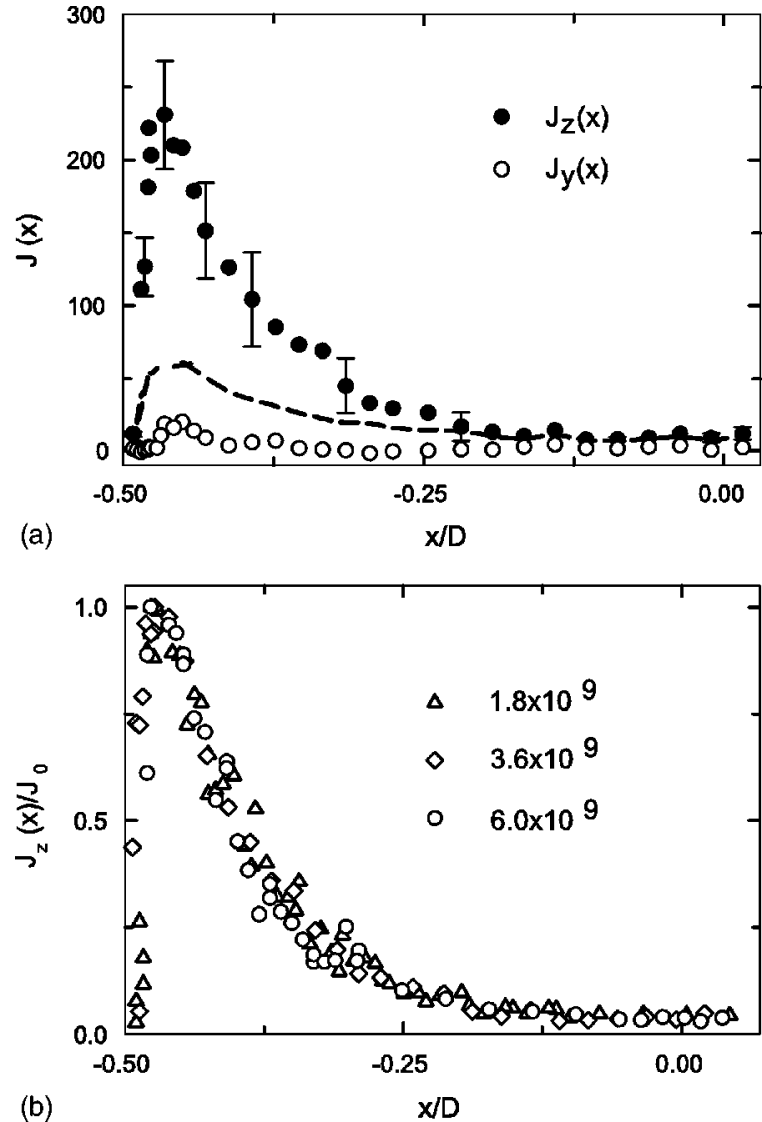

FIG. 4. (a) Measured vertical flux profile $J_{z}(x)$ (solid circles) and horizontal flux profile $J_{y}(x)$ (open circles) as a function of $x / D$ at $\mathrm{Ra}=3.6 \times 10^{9}$. The dashed curve shows the calculated $J_{z}(x)$ using the local average temperature $\bar{T}$ as the reference temperature (see text). (b) Normalized vertical flux profile $J_{z}(x) / J_{0}$ as a function of $x / D$ at $\mathrm{Ra}=1.8 \times 10^{9}$ (triangles), $3.6 \times 10^{9}$ (diamonds), and 6.0 $\times 10^{9}$ (circles). All the measurements are made in the $A=1$ cell.

pared with that in the central region. This is because the vertical velocity near the sidewall is larger than that near the cell center.

From the measured heat flux profile $J_{z}(x)$, one can estimate the total heat flux across the entire horizontal plan at the midheight of the cell. By assuming $J_{z}(x)$ is azimuthally symmetric, we have

$$
\widetilde{\mathrm{Nu}}=\frac{2}{(D / 2)^{2}} \int_{0}^{D / 2} J_{z}(x) x d x,
$$

where $D / 2$ is the radius of the convection cell. Using Eq. (3) and the measured heat flux profiles, we obtain $\widetilde{\mathrm{Nu}}$ for four different values of $\mathrm{Ra}$ studied in the experiment. Table I compares the calculated $\widetilde{\mathrm{Nu}}$ with the Nusselt number $\mathrm{Nu}$ measured in the $A=1$ cell [26] and in the $A=0.5$ cell $[16,29]$ both filled with water. It was found [26] that the measured $\mathrm{Nu}$ in the $A=1$ cell can be described by an effective power law $\mathrm{Nu}=0.19 \mathrm{Ra}^{0.28}$, which is used in Table I. The heat flux profile $J_{z}(x)$ shown in Fig. 4(a) contains several repeating measurements and thus is the most accurate one among the four profiles measured so far. One can estimate the error bar
TABLE I. Comparison between the calculated $\widetilde{\mathrm{Nu}}$ and the measured $\mathrm{Nu}$ in the $A=1$ cell (first three Rayleigh numbers) and in the $A=0.5$ cell (last Rayleigh number). The values of the measured $\mathrm{Nu}$ are taken from Ref. [26] ( $A=1$ cell) and Refs. [16,29] $(A=0.5$ cell).

\begin{tabular}{ccc}
\hline \hline $\mathrm{Ra}$ & Calculated $\tilde{\mathrm{Nu}}$ & Measured $\mathrm{Nu}$ \\
\hline $1.8 \times 10^{9}$ & 66 & 74 \\
$3.6 \times 10^{9}$ & 93 & 90 \\
$6.0 \times 10^{9}$ & 103 & 104 \\
$2.8 \times 10^{10}$ & 150 & 182 \\
\hline \hline
\end{tabular}

of $\widetilde{\mathrm{Nu}}$ using the experimental uncertainty of $\pm 0.05^{\circ} \mathrm{C}$ for $\delta T_{0}$ at each position. For $\mathrm{Ra}=3.6 \times 10^{9}$ we have $\delta \widetilde{\mathrm{Nu}} / \widetilde{\mathrm{Nu}} \simeq 0.14$, which is somewhat larger than the corresponding error bar for the measured $\mathrm{Nu}(<5 \%)$. It is seen from Table I that the relative difference between the calculated $\widetilde{\mathrm{Nu}}$ and the measured $\mathrm{Nu}$ in the $A=1$ cell is all within the estimated uncertainty. To further examine whether the heat flux profile $J_{z}(x)$ in a titled cylindrical cell is azimuthally symmetric, one needs to measure $J_{z}(x)$ in different azimuthal planes.

The dashed curve in Fig. 4(a) shows the calculated $J_{z}(x)$ when the local average temperature $\bar{T}$, instead of the most probable temperature $T_{0}$, is used as the reference temperature. In fact, one can decompose the temperature fluctuation $\delta T_{0}(\mathbf{r}, t)$ in Eq. (1) into two parts, $\delta T_{0}(\mathbf{r}, t)=[T(\mathbf{r}, t)-\bar{T}(\mathbf{r})]$ $+\left[\bar{T}(\mathbf{r})-T_{0}\right] \equiv \delta T(\mathbf{r}, t)+\left[\bar{T}(\mathbf{r})-T_{0}\right]$, where $\delta T$ is the temperature fluctuation relative to the local mean and $\left[\bar{T}(\mathbf{r})-T_{0}\right]$ is the time-averaged local temperature deviation from the common reference temperature $T_{0}$ [see Fig. 3(b)]. By further decomposing the local velocity as $v_{z}(\mathbf{r}, t)=\bar{v}_{z}(\mathbf{r})+\delta v_{z}(\mathbf{r}, t)$ and substituting these decompositions into Eq. (1), we have

$$
J_{z}(x)=\frac{\bar{v}_{z}(x)\left[\bar{T}(x)-T_{0}\right] H}{\kappa \Delta T}+\frac{\left\langle\delta v_{z}(x, t) \delta T(x, t)\right\rangle_{t} H}{\kappa \Delta T},
$$

where the first term $\bar{v}_{z}(x)\left[\bar{T}(x)-T_{0}\right] H /(\kappa \Delta T)$ is the contribution from the mean flow and the second term $\left\langle\delta v_{z}(x, t) \delta T(x, t)\right\rangle_{t} H /(\kappa \Delta T)$ results from the local velocity fluctuations. The dashed curve in Fig. 4(a) represents $\left\langle\delta v_{z}(x, t) \delta T(x, t)\right\rangle_{t} H /(\kappa \Delta T)$, because $\quad\left\langle\bar{v}_{z}(x) \delta T(x, t)\right\rangle_{t}=0$. Therefore, the difference between the solid circles and the dashed curve is $\bar{v}_{z}(x)\left[\bar{T}(x)-T_{0}\right] H /(\kappa \Delta T)$, which approaches zero in the central region of the cell. Near the sidewall, however, the contribution of $\bar{v}_{z}(x)\left[\bar{T}(x)-T_{0}\right] H /(\kappa \Delta T)$ can be as large as $73 \%$ of the total $J_{z}(x)$. It should be pointed out that both the mean flow and the velocity fluctuations are generated by the thermal plumes $[6,8,19,30]$. The decomposition of the local heat flux $J_{z}$ in Eq. (4) suggests that the Ra dependence of $J_{z}$ is determined by two different velocity scales and two temperature scales.

Figure 5(a) shows the measured vertical flux $J_{z}(z)$ (open circles) and two horizontal fluxes $J_{x}(z)$ (solid circles) and $J_{y}(z)$ (triangles) along the central axis of the cell. Contrary to the situation near the sidewall, heat transport near the center 


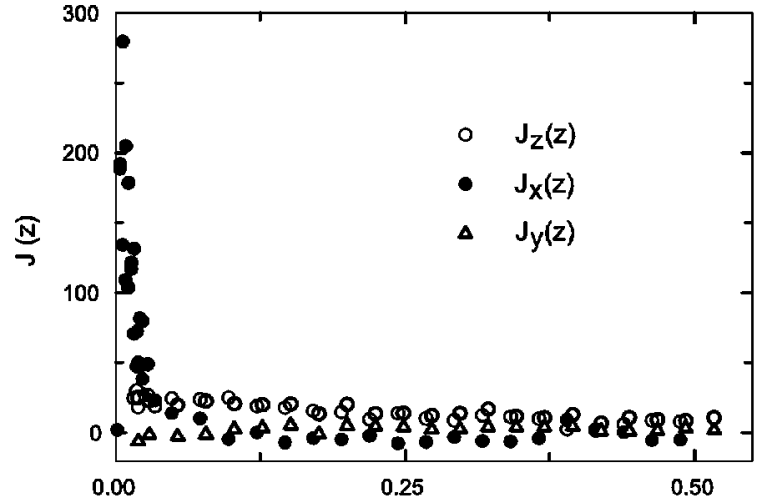

(a)

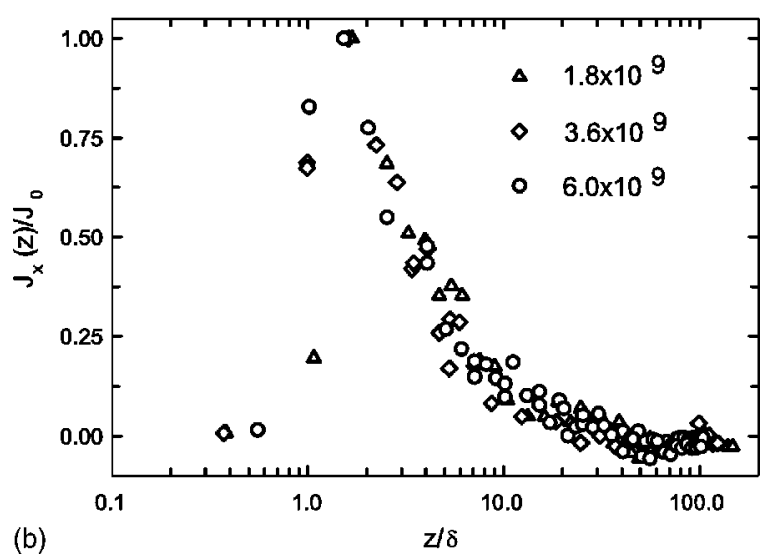

(b)

$\mathbf{z} / \delta$

FIG. 5. (a) Measured vertical flux profile $J_{z}(z)$ (open circles) and two horizontal flux profiles $J_{x}(z)$ (solid circles) and $J_{y}(z)$ (triangles) as a function of $z / H$. The measurements are made at $\mathrm{Ra}=3.6$ $\times 10^{9}$. (b) Normalized horizontal flux profile $J_{x}(z) / J_{0}$ as a function of $z / \delta$ at $\mathrm{Ra}=1.8 \times 10^{9}$ (triangles, $\delta=0.98 \mathrm{~mm}$ ), $3.6 \times 10^{9}$ (diamonds, $\delta=0.80 \mathrm{~mm}$ ), and $6.0 \times 10^{9}$ (circles, $\delta=0.69 \mathrm{~mm}$ ). All the measurements are made in the $A=1$ cell.

of the lower conducting surface is dominated by the horizontal flux along the direction of the LSC and the vertical flux is small. When compared with Fig. 4(a), we find that $J_{x}(z)$ peaks at a location much closer to the cell boundary and the width of the peak is narrower as well. The amplitude of the measured $J_{x}(z)$ is found to increase with Ra but its shape remains unchanged in the Ra range studied. This is clearly shown in Fig. 5(b). It is found that the peak position of the measured $J_{x}(z)$ scales with the thermal boundary layer thickness $\delta$. Lui and Xia [26] have shown that the measured $\delta$ at the cell center can be well described by an effective power law $\delta=425 \mathrm{Ra}^{-0.285} \mathrm{~mm}$. Figures 4 and 5 thus suggest that heat transport in the aspect-ratio- 1 cell is carried out primarily along the cell periphery in the direction of the LSC. It should be noted that, because the local convective heat transport over the conducting surface is not uniform, the heat flux profiles such as those shown in Fig. 5 may vary with the lateral position on the lower conducting surface.

\section{2. $A=0.5$ cell}

Figure 6(a) shows the measured vertical flux $J_{z}(x)$ (solid circles) and horizontal flux $J_{y}(x)$ (open circles) across the
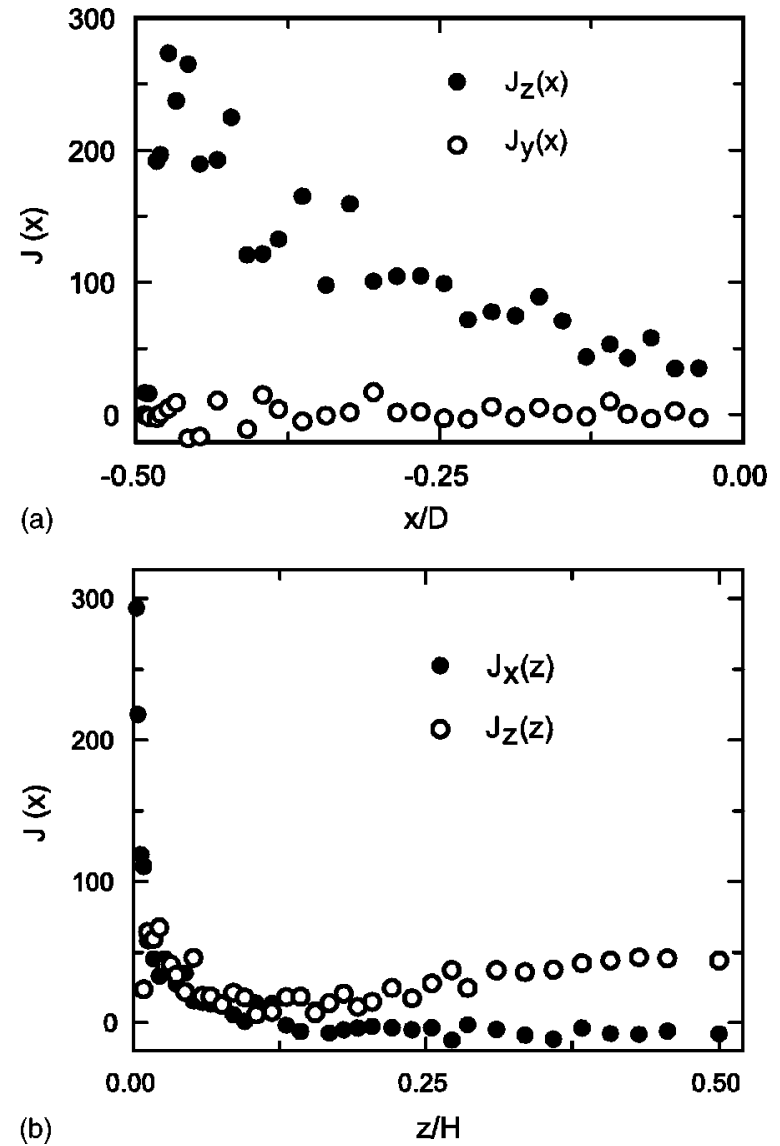

FIG. 6. (a) Measured vertical flux profile $J_{z}(x)$ (solid circles) and horizontal flux profile $J_{y}(x)$ (open circles) as a function of $x / D$. (b) Measured vertical flux profile $J_{z}(z)$ (open circles) and horizontal flux profile $J_{x}(z)$ (solid circles) as a function of $z / H$. All the measurements are made in the $A=0.5$ cell and at $\mathrm{Ra}=2.8 \times 10^{10}$.

cell diameter in the midplane of the cell. Figure 6(b) shows the measured $J_{z}(z)$ (open circles) and $J_{x}(z)$ (solid circles) along the central axis of the cell. It is seen that the main features of the heat flux profiles measured in the $A=0.5$ cell are similar to those in the $A=1$ cell. The vertical heat flux across the midplane of the cell is concentrated in the sidewall region and the horizontal heat flux is negligible. The convective heat transport near the center of the lower conducting surface is still dominated by the horizontal flux along the direction of the LSC and the vertical flux is small. The measured flux profiles in the $A=0.5$ cell show somewhat larger scatter, because the large-scale flow in the $A=0.5$ cell is not very stable and wobbles slowly in time even when the cell is tilted at a slightly larger angle [6]. As a result, the calculated $\widetilde{\mathrm{Nu}}$ using the measured $J_{z}(x)$ in Fig. 6(a) shows a larger deviation $(\sim 18 \%)$ from the measured $\mathrm{Nu}$ than those obtained in the $A=1$ cell (see Table I).

Figures 4-6 clearly show that the local convective heat flux in a closed cell is neither homogeneous nor isotropic. The spatial inhomogeneity and anisotropy are caused by the non uniform distribution of the thermal plumes in the cell. Our recent temperature and velocity measurements $[6,8]$ showed that the thermal plumes in a closed cell organize themselves in such a way that warm plumes accumulate on 
one side of the cell and cold plumes concentrate on the opposite side of the cell. The spatial separation of warm and cold plumes and the resulting LSC provide a fast channel along the cell periphery for the transport of heat. This physical picture explains why the plume-dominated sidewall region coincides with the "peak region," in which both $J_{z}(x)$ and $v_{z}$ reach maximum. Clearly, this is a self-organizing process in that the plume separation and LSC help each other and they are no longer independent of each other any more. Because velocity fluctuations in the central region are strong, most thermal plumes are mixed up in that region. Nevertheless, there are still some unmixed warm and cold plumes left, which give rise to a nonzero $J_{z}$ in the region.

\section{Fluctuations of the local convective heat flux}

In Eq. (1) we define the time-averaged local convective heat flux $\mathbf{J}(\mathbf{r})$. Using the simultaneous velocity and temperature data, we can also obtain the instantaneous convective heat flux

$$
\mathbf{j}(\mathbf{r}, t)=\frac{\left[\mathbf{v}(\mathbf{r}, t) \delta T_{0}(\mathbf{r}, t)\right] H}{\kappa \Delta T},
$$

with $\langle\mathbf{j}(\mathbf{r}, t)\rangle_{t}=\mathbf{J}(\mathbf{r})$. In calculating $j_{z}(\mathbf{r}, t)$, we use the convention that warm fluctuations $\left(\delta T_{0}>0\right)$ produce positive flux if their velocities are in the upward direction $\left(v_{z}>0\right)$. With this definition, both the rising warm plumes and the falling cold plumes contribute to a positive flux $j_{z}(\mathbf{r}, t)>0$. In this section, we discuss the statistical properties of the measured $j_{z}(\mathbf{r}, t)$ at three representative locations in the convection cell. As indicated in Fig. 1(b), the three locations are marked as position 1 (at the cell center), position 2 (near the sidewall), and position 3 (near the center of the lower conducting surface). Because the behavior of the flux fluctuations in the $A=0.5$ cell is found to be similar to that in the $A=1$ cell, we focus our attention hereinafter to the measurements in the $A=1$ cell.

\section{At the cell center}

Figure 7 shows the time series data for the temperature fluctuation $\delta T(t)$, horizontal velocity $v_{y}(t)$, vertical velocity $v_{z}(t)$, horizontal flux fluctuation $j_{y}(t)$, and vertical flux fluctuation $j_{z}(t)$ at the cell center (top to bottom curves). Temperature fluctuations at the cell center show irregular sharp spikes of variable heights. Many of the large spikes are produced by the thermal plumes passing through the temperature probe. They are symmetric relative to the local mean fluid temperature. The measured temperature histogram $H(\delta T)$ has a simple (symmetric) exponential form (not shown), which has been observed previously in other convection experiments [1,31]. The mean velocity at the cell center is zero, and fluctuations of both the horizontal and vertical velocity components are symmetric relative to the zero mean. The measured velocity histograms in both directions are found to have a Gaussian shape (not shown), which has also been observed in recent experiments $[6,19]$.

A key signature of the plume-generated temperature fluctuations is their correlation with the resulting vertical veloc-

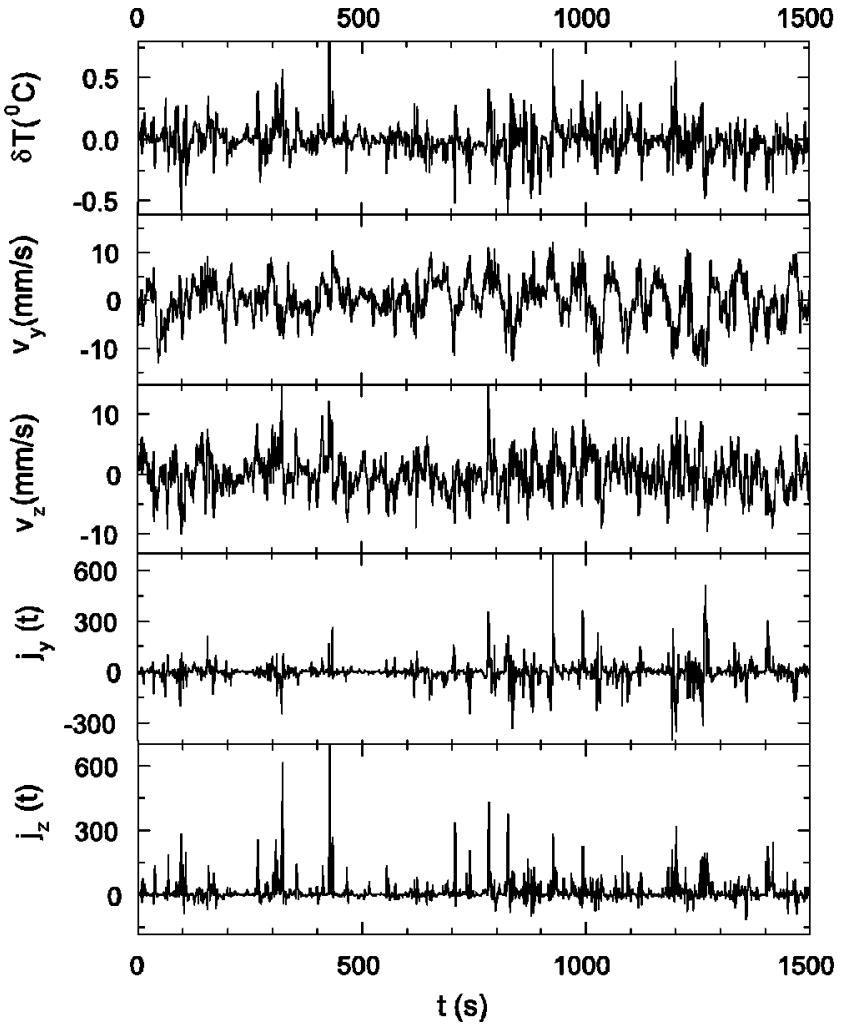

FIG. 7. Time series measurements of the temperature fluctuation $\delta T(t)$, horizontal velocity $v_{y}(t)$, vertical velocity $v_{z}(t)$, horizontal flux fluctuation $j_{y}(t)$, and vertical flux fluctuation $j_{z}(t)$ at the center of the $A=1$ cell (top to bottom curves). All the measurements are made at $\mathrm{Ra}=3.6 \times 10^{9}$.

ity fluctuations. This is because, when a cold plume falls (or a warm plume rises) through the central region, it always entrains the surrounding fluid in the downward (or upward) direction. Because both the cold and warm plumes produce positive fluxes in the vertical direction, the measured $j_{z}(\mathbf{r}, t)$ becomes highly skewed toward the positive direction, even though the temperature and velocity fluctuations themselves are symmetric. This is clearly shown in Fig. 7. The measured $j_{y}(\mathbf{r}, t)$, on the other hand, are symmetric relative to the zero mean, indicating that no such correlation exists between the temperature and horizontal velocity fluctuations. Figure 7 thus demonstrates that heat transport in turbulent convection is indeed driven by the bouyancy forces associated with the thermal plumes.

Figure 8(a) shows the measured histograms of the two horizontal flux fluctuations, $H\left(j_{x}\right) / H_{0}$ and $H\left(j_{y}\right) / H_{0}$ as functions of $j_{x} / \sigma_{x}$ and $j_{y} / \sigma_{y}$, respectively. To display the histograms measured at different values of Ra in the same graph, we normalize the histograms by their maximum value $H_{0}$, and the flux variables $j_{x}$ and $j_{y}$ are normalized by their standard deviations $\sigma_{x}$ and $\sigma_{y}$, respectively. It is seen that the measured $H\left(j_{y}\right)$ and $H\left(j_{x}\right)$ are symmetric and the mean values of $j_{x}$ and $j_{y}$ are zero $\left[J_{x}(\mathbf{r})=J_{y}(\mathbf{r})=0\right]$. The measured $H\left(j_{y}\right) / H_{0}$ and $H\left(j_{x}\right) / H_{0}$ for different values of Ra can all be brought into coincidence, once $j_{y}$ and $j_{x}$ are scaled by their rms values. Plots of $H\left(j_{x}\right) / H_{0}$ vs $j_{x} / \sigma_{x}$ [and $H\left(j_{y}\right) / H_{0}$ vs $\left.j_{y} / \sigma_{x}\right]$ remain unchanged in the Ra range studied and only $\sigma_{x}$ 

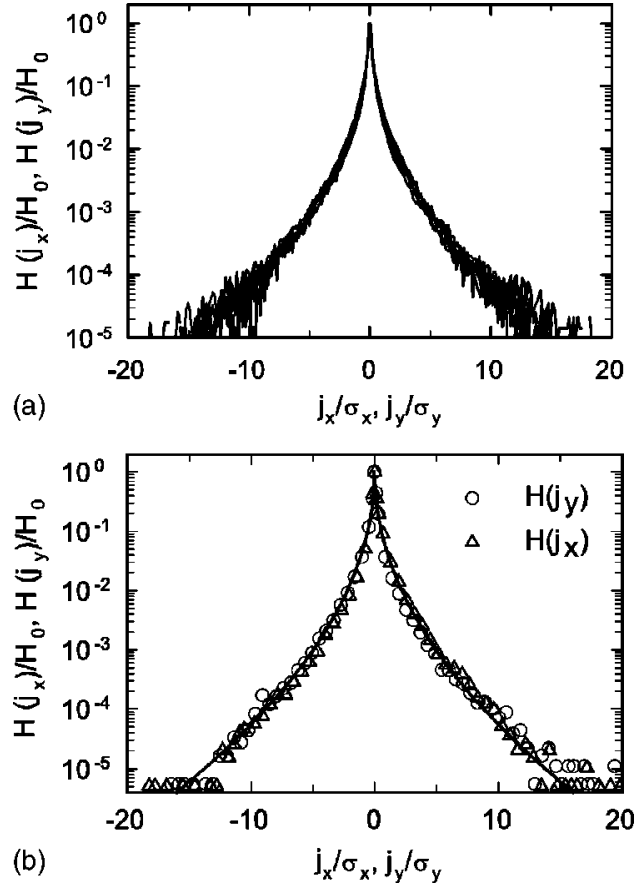

FIG. 8. (a) Measured histograms of the two horizontal flux fluctuations $H\left(j_{y}\right) / H_{0}$ and $H\left(j_{x}\right) / H_{0}$ as functions of $j_{x} / \sigma_{x}$ and $j_{y} / \sigma_{y}$, respectively. All the measurements are made at the center of the $A=1$ cell. The values of $\mathrm{Ra}$ are $9.8 \times 10^{8}, 1.8 \times 10^{9}, 2.6 \times 10^{9}, 3.6$ $\times 10^{9}$, and $6.0 \times 10^{9}$ (eight curves in total). (b) Measured histograms $H\left(j_{y}\right) / H_{0}$ (open circles) and $H\left(j_{x}\right) / H_{0}$ (open triangles) at $\mathrm{Ra}=3.6 \times 10^{9}$. The solid curve shows the fitted function $H(j) / H_{0}$ $=\exp \left[-\alpha(j / \sigma)^{\beta}\right]$, with $\alpha=3.1$ and $\beta=0.5$ ( $\sigma$ is a known parameter).

(and $\sigma_{y}$ ) changes with $\mathrm{Ra}$. The measured histograms have a universal form, which can be described by a stretched exponential function $H(j) / H_{0}=\exp \left[-\alpha(j / \sigma)^{\beta}\right]$, with $\alpha=3.1$ and $\beta=0.5$. The solid curve in Fig. 8 (b) shows the fit. The fact that the measured $H\left(j_{x}\right) / H_{0}$ and $H\left(j_{y}\right) / H_{0}$ have the same shape suggests that fluctuations of the local heat flux in the two horizontal directions are statistically the same.

Figure 9 shows the measured histograms of the vertical flux fluctuation $H\left(j_{z}\right) / H_{0}$ as a function of $j_{z} / \sigma_{z}$ for different values of Ra. Again, $H\left(j_{z}\right) / H_{0}$ in the Ra range studied has the

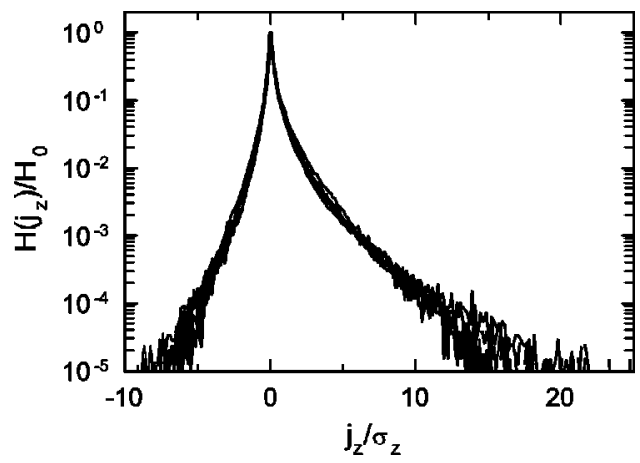

FIG. 9. Measured histograms of the vertical flux fluctuation $H\left(j_{z}\right) / H_{0}$ as a function of $j_{z} / \sigma_{z}$ at the center of the $A=1$ cell. The values of $\mathrm{Ra}$ are $1.8 \times 10^{9}, 2.6 \times 10^{9}, 3.6 \times 10^{9}, 4.8 \times 10^{9}, 6.0$ $\times 10^{9}$, and $7.6 \times 10^{9}$ ( six curves in total).

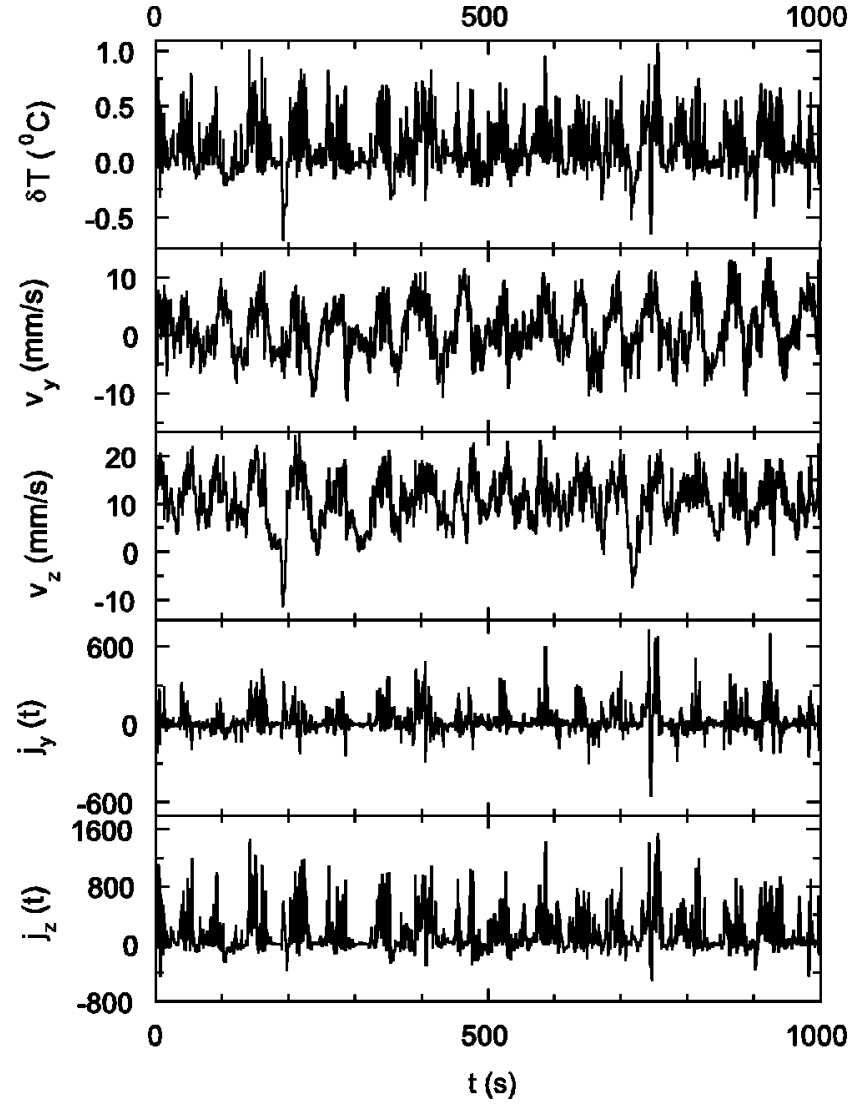

FIG. 10. Time series measurements of the temperature fluctuation $\delta T(t)$, horizontal velocity $v_{y}(t)$, vertical velocity $v_{z}(t)$, horizontal flux fluctuation $j_{y}(t)$, and vertical flux fluctuation $j_{z}(t)$ near the sidewall of the $A=1$ cell (top to bottom curves). All the measurements are made at $\mathrm{Ra}=3.6 \times 10^{9}$.

same shape, once $j_{z}$ is scaled by its rms value $\sigma_{z}$. An important difference between the horizontal and vertical flux fluctuations is that fluctuations of the vertical heat flux are asymmetric, with more fluctuations in the positive direction. The net gain of the positive fluctuations gives rise to a small mean value for the vertical heat flux at the cell center. It is seen that the asymmetry occurs mainly in the tail part of $H\left(j_{z}\right) / H_{0}$. As discussed above, this asymmetry is caused by the correlated temperature and velocity signals, which are generated by a small number of energetic warm and cold plumes remaining in the central region.

\section{Near the sidewall}

Figure 10 shows the time series data for the temperature fluctuation $\delta T(t)$, horizontal velocity $v_{y}(t)$, vertical velocity $v_{z}(t)$, horizontal flux fluctuation $j_{y}(t)$, and vertical flux fluctuation $j_{z}(t)$ near the sidewall (top to bottom curves). The measurements are made at position 2 shown in Fig. 1(b), which is in the midplane of the cell and $9 \mathrm{~mm}$ away from the sidewall. This is the position at which the measured $J_{z}(x)$ reaches maximum [see Fig. 4(a)]. In contrast to the situation at the cell center, temperature fluctuations near the sidewall are highly skewed toward one direction. Most of the large upward going spikes are associated with the rising warm 

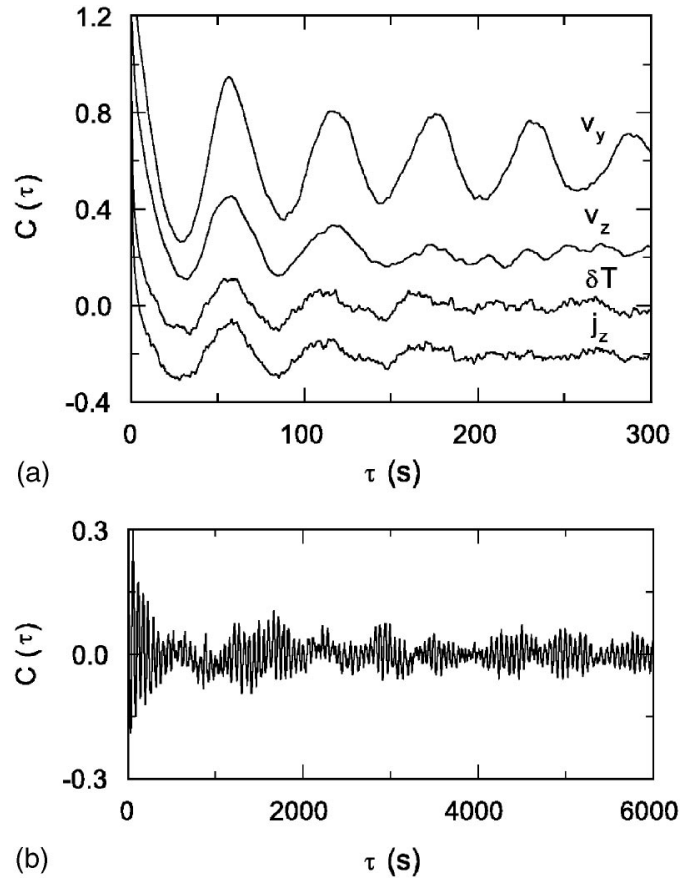

FIG. 11. (a) Measured autocorrelation function $C(\tau)$ for the horizontal velocity $v_{y}(t)$, vertical velocity $v_{z}(t)$, temperature fluctuation $\delta T(t)$, and vertical flux fluctuation $j_{z}(t)$ (top to bottom curves). The measurements are made near the sidewall of the $A=1$ cell and at $\mathrm{Ra}=3.6 \times 10^{9}$. For clarity, the origin of the vertical axis for the top two curves and the bottom one is shifted by an amount of 0.6 , 0.2 , and -0.2 , respectively. (b) Measured $C(\tau)$ for the horizontal velocity $v_{y}(t)$ over longer delay time $\tau$. The experimental conditions remain the same as the above.

plumes in the region. It is seen that the thermal plumes do not arrive randomly; rather, they arrive in groups with a welldefined frequency. Qiu and Tong $[7,8]$ have shown that such an oscillation is caused by the alternating emission of cold and warm plumes between the upper and lower boundary layers. When they move up in the sidewall region, the warm plumes entrain the surrounding fluid and produce a mean flow in the vertical direction. It is seen from Fig. 10 that the resulting vertical velocity $v_{z}$ follows the temperature oscillation well. It is also seen that the horizontal velocity $v_{y}$ has a zero mean value and oscillates at the same frequency as that of the temperature oscillation [19]. A similar oscillation is also observed in the measured vertical flux fluctuation $j_{z}(t)$.

Figure 11(a) shows the measured flux autocorrelation function $C(\tau)=\left\langle j_{z}(t) j_{z}(t+\tau)\right\rangle /\left(j_{z}\right)_{r m s}^{2}$ as a function of delay time $\tau$ (bottom curve). For comparison, we also display the autocorrelation functions of the two velocity components $v_{y}(t)$ and $v_{z}(t)$ and the temperature fluctuation $\delta T$ in Fig. 11(a). The measured correlation functions share some common features but also show differences. First, there exists a well-defined oscillation frequency, which is the same for all the correlation functions. Second, the oscillation amplitude of the correlation functions changes with delay time $\tau$. For the flux and temperature autocorrelation functions, the oscillation amplitude decays with time, indicating that the temperature oscillation has a finite coherence time $\tau_{c}[7,8]$. The value of $\tau_{c}$ can be obtained simply by counting how many

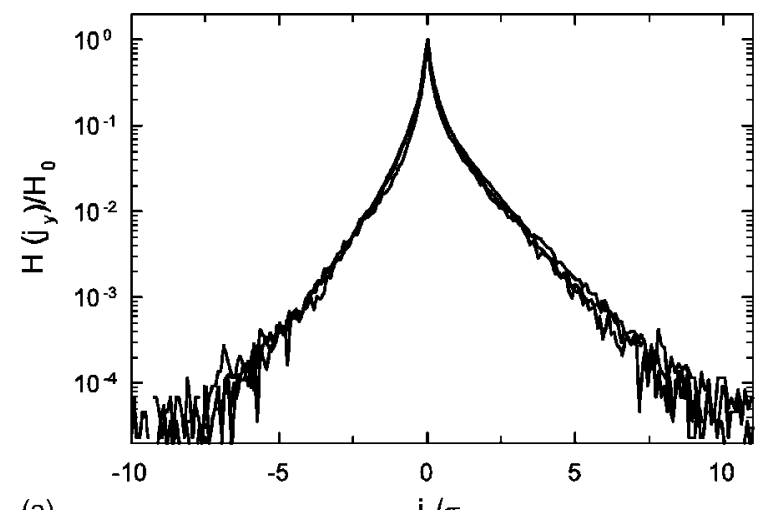

(a)

$\mathrm{j}_{\mathrm{y}} / \sigma_{\mathrm{y}}$

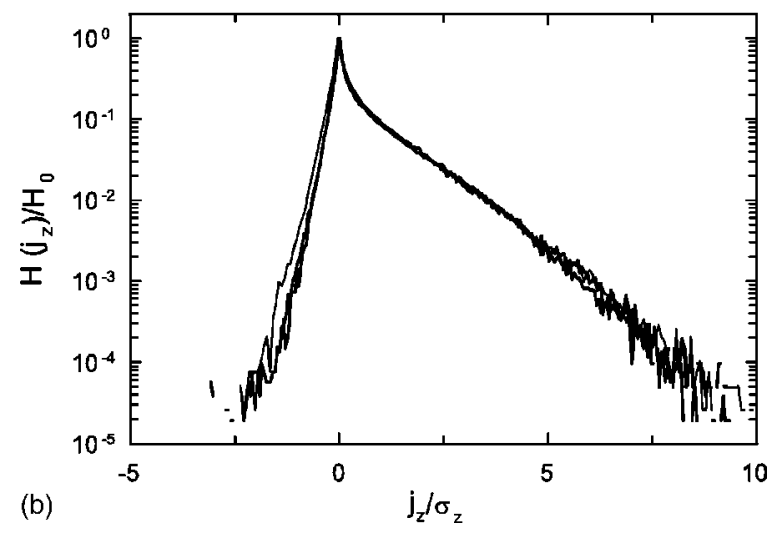

FIG. 12. (a) Measured histograms of the horizontal flux fluctuation $H\left(j_{y}\right) / H_{0}$ as a function of $j_{y} / \sigma_{y}$. (b) Measured histograms of the vertical flux fluctuation $H\left(j_{z}\right) / H_{0}$ as a function of $j_{z} / \sigma_{z}$. All the measurements are made near the sidewall of the $A=1$ cell. The values of Ra are $1.8 \times 10^{9}, 3.6 \times 10^{9}$, and $6.0 \times 10^{9}$.

oscillation cycles are in the measured $C(\tau)$. The velocity oscillations, on the other hand, have a much longer coherence time. Figure 11(b) displays the measured $C(\tau)$ for $v_{y}(t)$ over longer delay time $\tau$. It is seen that the oscillation amplitude decays in the first $500 \mathrm{~s}$ or so and then continues to oscillate almost indefinitely. The autocorrelation function for $v_{z}(t)$ (not shown) shows a similar behavior. Funfschilling and Ahlers [32] reported recently that the velocity autocorrelation function near the conducting surface exhibits the similar behavior. The slowly varying amplitude modulation, as shown in Fig. 11(b), suggests that the velocity oscillation has a narrow bandwidth, which produces frequency beating [33]. It is clearly seen from Figs. 10 and 11 that the measured $j_{z}(t)$ oscillates with the same frequency and phase as those of the temperature fluctuations. The two figures thus further demonstrate that the local heat transport in turbulent convection over the parameter range explored is indeed carried out by the thermal plumes. The oscillatory behavior of the local heat flux is not observed in the global measurement of the Nusselt number, as the spatial averaging over the entire convecting fluid can smooth out the local flux variations.

Figures 12(a) and 12(b) show, respectively, the normalized histograms of the horizontal flux $j_{y}$ and vertical flux $j_{z}$ near the sidewall. Similar to the situation at the cell center, the measured histograms for different values of Ra can all be brought into coincidence, once $j_{y}$ and $j_{z}$ are scaled, respec- 


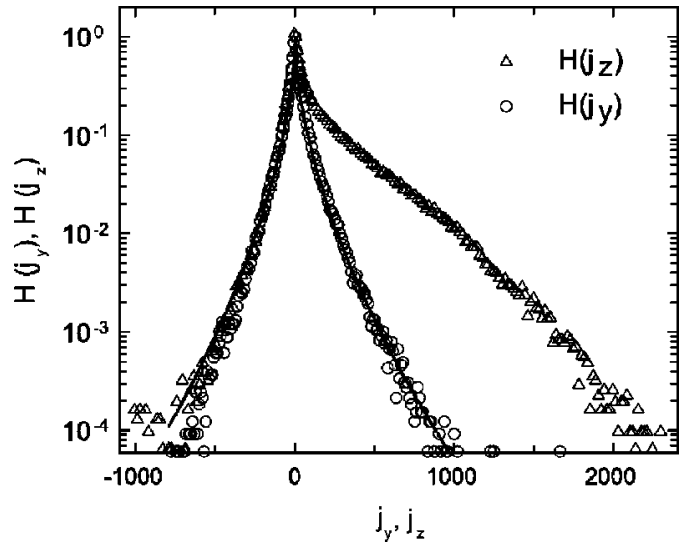

FIG. 13. Comparison between the vertical flux histogram $H\left(j_{z}\right)$ (open triangles) and the horizontal flux histogram $H\left(j_{y}\right)$ (open circles) near the sidewall. The measurements are made at $\mathrm{Ra}=3.6$ $\times 10^{9}$. The solid curve shows a fit to the open circles and the fitted function is $H\left(j_{y}\right) / H_{0}=\exp \left[-\alpha\left(j_{y} / \sigma_{y}\right)^{\beta}\right]$ with $\alpha=2.42$ and $\beta=0.58$ ( $\sigma_{y}=88$ is a known parameter).

tively, by their rms values $\sigma_{y}$ and $\sigma_{z}$. Plots of $H\left(j_{y}\right) / H_{0}$ vs $j_{y} / \sigma_{y}$ [and $H\left(j_{z}\right) / H_{0}$ vs $j_{z} / \sigma_{z}$ ] remain unchanged in the Ra range studied and only $\sigma_{y}$ (and $\sigma_{z}$ ) changes with $\mathrm{Ra}$. It is seen from Fig. 12 that fluctuations of the horizontal flux $j_{y}$ (out of the rotation plane of the LSC) are symmetric and their mean value is approximately zero. Fluctuations of the vertical flux $j_{z}$, on the other hand, are highly skewed toward the positive direction. The net gain of the positive fluctuations gives rise to a large mean vertical heat flux near the sidewall. It is also seen that the measured $H\left(j_{z}\right) / H_{0}$ has a long decay tail, indicating that the flux fluctuations produced by the thermal plumes contain some large but rare events.

In Fig. 13 we compare the vertical flux histogram $H\left(j_{z}\right)$ (open triangles) with the horizontal flux histogram $H\left(j_{y}\right)$ (open circles) measured at $\mathrm{Ra}=3.6 \times 10^{9}$. It is seen that negative flux fluctuations in the two directions are approximately the same, whereas positive flux fluctuations in the vertical direction (parallel to gravity) are much larger than those in the horizontal direction. Figure 13 suggests that the measured $j_{z}$ contains both the correlated (active) and uncorrelated (passive) fluctuations. The negative fluctuations together with their symmetric counterpart of small positive fluctuations are produced by uncorrelated temperature and velocity signals. These fluctuations are approximately the same as those in the horizontal direction and do not contribute to the local heat transport. Similar to the situation at the cell center, the measured $H\left(j_{y}\right)$ near the sidewall can also be described by a stretched exponential function $H\left(j_{y}\right) / H_{0}$ $=\exp \left[-\alpha\left(j_{y} / \sigma_{y}\right)^{\beta}\right]$. The solid curve in Fig. 13 shows the fit with $\alpha=2.42$ and $\beta=0.58$ ( $\sigma_{y}=88$ is a known parameter). The value of $\beta$ obtained near the sidewall is slightly larger than that obtained in the central region.

The correlated temperature and velocity signals are generated by the thermal plumes and produce large positive fluctuations of $j_{z}$, which give rise to a large mean value of the vertical heat flux. It is seen from Fig. 10 that the instantaneous vertical flux $j_{z}$ near the sidewall (bottom curve) is highly skewed with many upward going spikes superposed

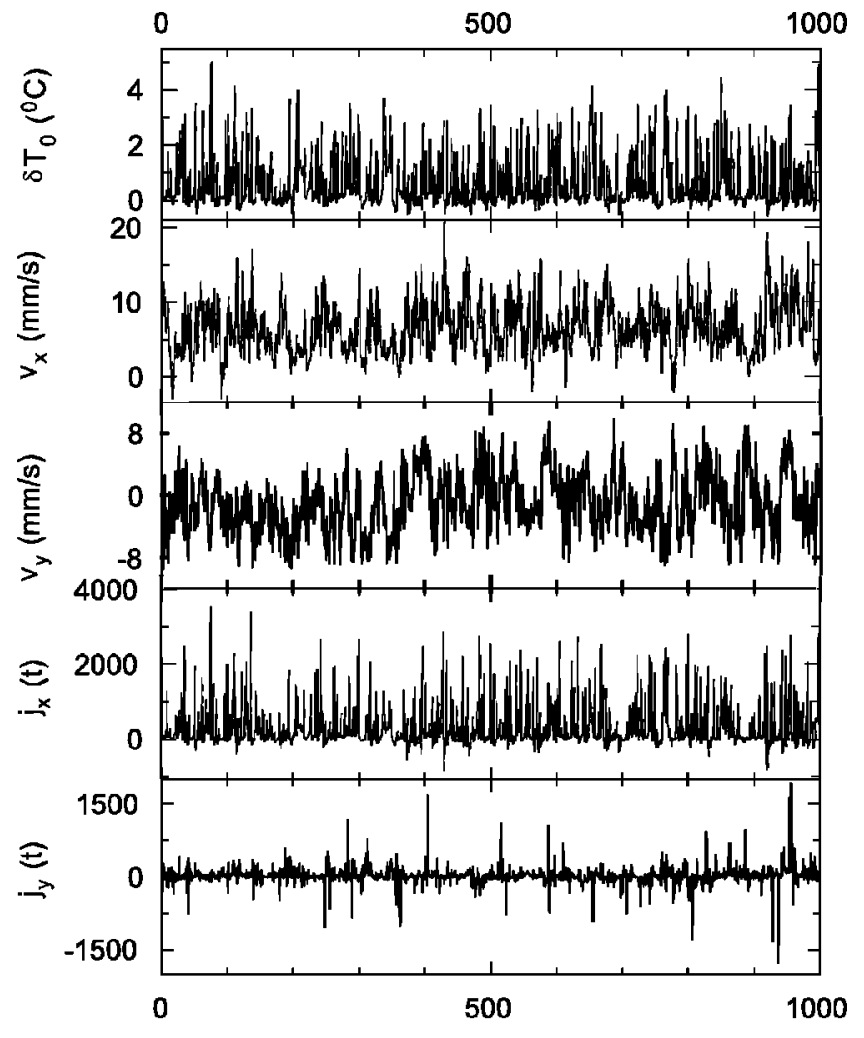

$t(s)$

FIG. 14. Time series measurements of the temperature fluctuation $\delta T(t)$, two horizontal velocity components $v_{x}(t)$ and $v_{y}(t)$, and two horizontal flux fluctuations $j_{x}(t)$ and $j_{y}(t)$ near the lower conducting surface of the $A=1$ cell (top to bottom curves). All the measurements are made at $\mathrm{Ra}=3.6 \times 10^{9}$.

on an average base line. Most of the large spikes are associated with the rising warm plumes at position 2 . While the input heating power is constant (at a fixed $\mathrm{Ra}$ ) and uniform across the entire conducting surface, the release of this power to the convecting fluid via $j_{z}$ is often concentrated in small time intervals or in localized regions. Near the sidewall the most probable events, defined by $\left|j_{z} / \sigma_{z}\right| \leqslant 0.1$, account for only $\sim 36 \%$ of the total population. In the central region, however, this number increases to $\sim 50 \%$. It is seen from Fig. 13 that some rare fluctuations (produced by the energetic plumes) can carry an instantaneous heat flux as large as 2000. This value is more than 20 times larger than the average heating flux $(\mathrm{Nu} \simeq 93)$. The measured $H\left(j_{z}\right)$ is found to be highly non-Gaussian and exhibits an exponential-like tail.

\section{Near the lower conducting surface}

Figure 14 shows the time series data for the temperature fluctuation $\delta T(t)$, two horizontal velocity components $v_{x}(t)$ and $v_{y}(t)$, and two horizontal flux fluctuations $j_{x}(t)$ and $j_{y}(t)$ near the lower conducting surface (top to bottom curves). The measurements are made at position 3 shown in Fig. 1(b), which is at $z=1.1 \mathrm{~mm}$ above the center of the lower conducting plate. This is the position at which the measured $J_{x}(z)$ reaches maximum. As shown in Fig. 5(b), the peak position of the measured $J_{x}(z)$ scales with the thermal boundary layer 


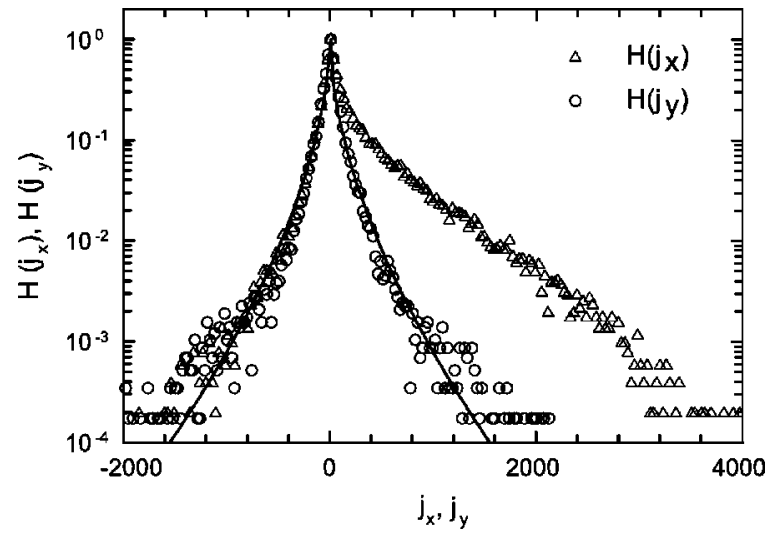

FIG. 15. Comparison between the two horizontal flux histograms $H\left(j_{x}\right)$ (open triangles) and $H\left(j_{y}\right)$ (open circles) near the lower conducting surface at $\mathrm{Ra}=3.6 \times 10^{9}$. The solid curve shows a fit to the open circles and the fitted function is $H\left(j_{y}\right) / H_{0}=\exp$ $\times\left[-\alpha\left(j_{y} / \sigma_{y}\right)^{\beta}\right]$ with $\alpha=2.59$ and $\beta=0.58\left(\sigma_{y}=174\right.$ is a known parameter).

thickness $\delta$, and the flux profile decays quickly to zero when $z$ is moved away from the bottom plate. Similar to the situation near the sidewall, temperature fluctuations near the bottom plate are highly skewed toward the positive direction, indicating that there are many warm plumes erupting from the lower thermal boundary layer. It is found that the amplitude of the temperature fluctuations near the bottom plate is more than four times larger than that near the sidewall (see Fig. 10). As they travel from the bottom plate to the sidewall region, the warm plumes lose their potential energy (proportional to $\delta T$ ) but gain kinetic energy (which is proportional to $v_{z}^{2}$ ). The velocity field near the bottom plate differs from that near the sidewall [6]. It is seen that the dominant mean flow near the bottom plate is in the $x$ direction, whereas the mean value of the other two velocity components $v_{y}$ and $v_{z}$ (not shown) is very small. In fact, the horizontal mean flow $\left(v_{x}\right)$ near the bottom plate is a part of the LSC, which spans the height of the convection cell. It is seen from Fig. 14 that it is this large-scale circulation which correlates with the temperature fluctuations produced by the thermal plumes. The strong correlation gives rise to a large positive heat flux $\left(j_{x}\right)$ along the direction of the LSC. It is found that the typical amplitude of $j_{x}$ near the bottom plate is more than two times larger than that of $j_{z}$ near the sidewall (see Fig. 10).

Figure 15 compares the two horizontal flux histograms $H\left(j_{x}\right)$ (open triangles) and $H\left(j_{y}\right)$ (open circles), measured at $\mathrm{Ra}=3.6 \times 10^{9}$. It is seen that the measured $j_{y}$ fluctuates symmetrically and its mean value is approximately zero. Similar to the situation near the sidewall, the measured $H\left(j_{y}\right)$ near the bottom plate can also be described by a stretched exponential function, $H\left(j_{y}\right) / H_{0}=\exp \left[-\alpha\left(j_{y} / \sigma_{y}\right)^{\beta}\right]$. The solid curve in Fig. 15 shows the fit with $\alpha=2.59$ and $\beta=0.58$ ( $\sigma_{y}=174$ is a known parameter). The value of $\beta$ obtained near the bottom plate is the same as that obtained near the sidewall. Fluctuations of $j_{x}$, on the other hand, are highly skewed toward the positive direction, giving rise to a net mean flux in the $x$ direction. It is seen that negative flux fluctuations in the two horizontal directions are approxi- mately the same, whereas positive flux fluctuations along the direction of the LSC are much larger than those in the $y$ direction. Figure 15 thus further demonstrates that the large positive flux fluctuations result from the correlated temperature and velocity signals, which are generated by the thermal plumes. By comparing Fig. 15 with Fig. 13, we find that both the correlated $\left(+j_{x}\right)$ and uncorrelated $\left(j_{y}\right)$ flux fluctuations near the lower conducting surface are approximately twice as large as those near the sidewall.

\section{SUMMARY}

We have carried out a systematic study of local convective heat transport in turbulent thermal convection. The local convective heat flux is obtained from simultaneous measurements of the local velocity and temperature in two cylindrical cells filled with water. The measurements were conducted at a fixed Prandtl number $(\operatorname{Pr} \simeq 5.5)$ and over varying Rayleigh numbers $\left(10^{9} \leqslant \mathrm{Ra} \leqslant 3 \times 10^{10}\right)$ and spatial positions across the entire convection cell. From the spatial measurements of the time-averaged local convective heat flux, we obtain an interesting physical picture of the dynamics in turbulent convection. It is found that the local convective heat transport in turbulent convection over the parameter range studied is carried out primarily by the thermal plumes. The thermal plumes organize themselves in a closed cell in such a way that warm plumes accumulate on one side of the cell and cold plumes concentrate on the opposite side of the cell. The spatial separation of warm and cold plumes and the resulting large-scale circulation provide a fast channel along the cell periphery for the transport of heat. In the central region, velocity fluctuations are large, and most thermal plumes are mixed up. Nevertheless, there still exist some unmixed thermal plumes in the region, which give rise to a small convective heat flux in the vertical direction. The experiment reveals that the nonuniform spatial distribution of the local convective heat flux is generated by a unique distribution of the thermal plumes in a closed cell and thus settles a long-debated issue on how heat is transported in small-aspect-ratio cells [1-4].

With the simultaneous velocity and temperature data, we have also obtained the instantaneous local convective heat flux $\mathbf{j}(\mathbf{r}, t)$ and studied its statistical properties at three representative locations in the cell. A careful comparison between the local flux fluctuations near the conducting plate and near the sidewall provides important insights into the dynamic evolution of the thermal plumes, as they travel along the large-scale circulation. It is found that the measured $\mathbf{j}(\mathbf{r}, t)$ contains both uncorrelated (passive) and correlated (active) fluctuations. The former are symmetric fluctuations produced by uncorrelated temperature and velocity signals (turbulent background) and do not contribute to the average value of the local convective heat flux. These fluctuations are approximately isotropic and their histograms have a common form, which can be described by a stretched exponential function, $H(j)=H_{0} \exp \left[-\alpha(j / \sigma)^{\beta}\right]$. The value of $\beta$ obtained in the central region is 0.5 and that obtained in the plume-dominated region near the sidewall and near the bottom plate is 0.58 . The latter are large positive fluctuations 
produced by the correlated temperature and velocity signals. They arise only in the vertical direction parallel to gravity and are associated with the thermal plumes in the region. These fluctuations give rise to a large mean value of the vertical heat flux. It is found that the large positive flux fluctuations occur more often in the plume-dominated region (near the sidewall and near the conducting surface) and their histograms exhibit an exponential-like tail. Some rare fluctuations produced by the energetic plumes in the region can carry an instantaneous heat flux 20 times larger than the mean heating flux.

\section{ACKNOWLEDGMENTS}

We thank E. Ching and D. Lohse for useful discussions. The assistance of M. Lucas and his team in fabricating the convection cells is gratefully acknowledged. P. T. was supported in part by the National Science Foundation under Grant No. DMR-0071323 and by the Research Grants Council of Hong Kong SAR under Grant No. HKUST603003. K.-Q. X. was supported by the Research Grants Council of Hong Kong SAR under Grant No. CUHK403003.
[1] B. Castaing, G. Gunaratne, F. Heslot, L. Kadanoff, A. Libchaber, S. Thomae, X.-Z. Wu, S. Zaleski, and G. Zanetti, J. Fluid Mech. 204, 1 (1989).

[2] E. Siggia, Annu. Rev. Fluid Mech. 26, 137 (1994).

[3] S. Grossmann and D. Lohse, J. Fluid Mech. 407, 27 (2000).

[4] L. P. Kadanoff, Phys. Today 54 No. 8, 34 (2001).

[5] X.-L. Qiu, S. H. Yao, and P. Tong, Phys. Rev. E 61, R6075 (2000).

[6] X.-L. Qiu and P. Tong Phys. Rev. E 64, 036304 (2001).

[7] X.-L. Qiu and P. Tong, Phys. Rev. Lett. 87, 094501 (2001).

[8] X.-L. Qiu and P. Tong, Phys. Rev. E 66, 026308 (2002).

[9] S.-Q. Zhou and K.-Q. Xia, Phys. Rev. Lett. 89, 184502 (2002).

[10] X. Chavanne, F. Chilla, B. Castaing, B. Hebral, B. Chabaud, and J. Chaussy, Phys. Rev. Lett. 79, 3648 (1997).

[11] X. Chavanne, F. Chilla, B. Chabaud, B. Castaing and B. Hebral, Phys. Fluids 13, 1300 (2001).

[12] J. J. Niemela, L. Skrbek, K. R. Sreenivasan, and R. J. Donnelly, Nature (London) 404, 837 (2000).

[13] X. Xu, K. M. Bajaj, and G. Ahlers, Phys. Rev. Lett. 84, 4357 (2000).

[14] G. Ahlers and X. Xu, Phys. Rev. Lett. 86, 3320 (2001).

[15] K.-Q. Xia, S. Lam, and S.-Q. Zhou, Phys. Rev. Lett. 88, 064501 (2002).

[16] Y.-B. Du and P. Tong, J. Fluid Mech. 407, 57 (2000).

[17] H. Tennekes and J. L. Lumley, A First Course in Turbulence (MIT Press, Cambridge, MA, 1972).

[18] A. Belmonte, A. Tilgner, and A. Libchaber, Phys. Rev. E 50, 269 (1994)

[19] X.-L. Qiu, X.-D. Shang, P. Tong, and K.-Q. Xia, Phys. Fluids 16, 412 (2004).
[20] CRC Handbook of Chemistry and Physics, 74th ed., edited by D. R. Lide (CRC Press, Boca Raton, FL, 1993).

[21] L. E. Drain, The Laser Doppler Technique (Wiley \& Sons, New York, 1980).

[22] S. Cioni, S. Ciliberto, and J. Sommeria, J. Fluid Mech. 335, 111 (1997).

[23] J. J. Niemela, L. Skrbek, K. R. Sreenivasan, and R. J. Donnelly, J. Fluid Mech. 449, 169 (2001).

[24] K. R. Sreenivasan, A. Bershadskii, and J. J. Niemela, Phys. Rev. E 65, 056306 (2002).

[25] S. Ciliberto, S. Cioni, and C. Laroche, Phys. Rev. E 54, R5901 (1996).

[26] S.-L. Lui and K.-Q. Xia, Phys. Rev. E 57, 5494 (1998).

[27] Because of the spatial variation of the measured $\delta$ over the conducting surface (see Ref. [26] for more details), the pointwise diffusive heat flux $\left.\left(J_{d}\right)_{z}\right|_{\text {wall }}$ varies with the lateral position. The spatial average of $\left.\left(J_{d}\right)_{z}\right|_{\text {wall }}$ over the whole conducting surface should be equal to the measured Nusselt number $\mathrm{Nu}$.

[28] X.-D. Shang, X.-L. Qiu, P. Tong, and K.-Q. Xia, Phys. Rev. Lett. 90, 074501 (2003).

[29] Y.-B. Xin and K.-Q. Xia, Phys. Rev. E 56, 3010 (1997).

[30] H.-D. Xi, S. Lam, and K.-Q. Xia, J. Fluid Mech. 503, 47 (2004)

[31] Y.-B. Du and P. Tong, Phys. Rev. E 63, 046303 (2001).

[32] D. Funfschilling and G. Ahlers, Phys. Rev. Lett. 92, 194502 (2004).

[33] J. S. Bendat and A. G. Piersol, Engineering Applications of Correlation and Spectral Analysis, 2nd ed. (Wiley \& Sons, New York, 1993), Chap. 6. 